\title{
Radiology and Imaging Technology
}

\section{Characterization of the Olfactory Pathway by Anisotropic Diffu- sion Using Nuclear Magnetic Resonance Imaging in a Pediatric Population}

\author{
Héctor Ramírez-Flores ${ }^{1}$, Eduardo Barragan-Perez ${ }^{2}$, Pilar Dies-Suarez ${ }^{2}$, Diana Platas-Neri ${ }^{3}$, Julio \\ Garcia $^{4}$ and Silvia Hidalgo-Tobón ${ }^{1,2^{*}}$
}

\author{
${ }^{1}$ Physics Department, Universidad Autónoma Metropolitana Unidad Iztapalapa, México \\ ${ }^{2}$ Imagenology Department, Hospital Infantil de México Federico Gómez, México \\ ${ }^{3}$ Center of Research in Cognitive Sciences, Universidad Autónoma del Estado de Morelos, México \\ ${ }^{4}$ Cumming School of Medicine, University of Calgary, Canada
}

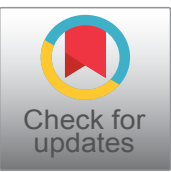

*Corresponding author: Dr. Silvia Hidalgo-Tobón, Radiology Department, Hospital Infantil de México Federico Gómez, Dr. Márquez 162, Doctores, 06720 Ciudad de México, CDMX, México; Physics Department, Universidad Autónoma Metropolitana Unidad Iztapalapa, Av. San Rafael Atlixco 186, Leyes de Reforma 1ra Secc, 09340 Ciudad de México, CDMX, México, Tel: +(52)55-5588-9523

\begin{abstract}
Objective: To characterize white matter tracts of the olfactory system and evaluate differences in diffusion parameters as a function of age in healthy children.

Introduction: One of the techniques of Nuclear Magnetic Resonance is Diffusion Tensor Imaging, which measures the speed of diffusion of extracellular water molecules found in tissues. Diffusion tensor imaging allows virtual dissections of functional white matter tracts in the human brain in vivo using regions of interest (ROI) [1]. The intrinsic trajectories of the olfactory pathway that can be identified using the diffusion-weighted magnetic resonance technique provide an important anatomical reference for the evaluation of clinical disorders commonly associated with the olfactory system in the brains of pediatric patients [2].

Materials and methods: Diffusion tensor imaging was performed in fifty-one volunteer children, aged 6-16 years. The study was conducted with the consent of their parents. Generalized diffusion parameters such as Fractional Anisotropy (FA) and Apparent Diffusion Coefficient (ADC) of the total tracts of white matter were correlated with age using a Pearson correlation.

Results: A significant negative correlation between ADC values and age was obtained. A sufficiently significant correlation of the generalized FA values was not found over the white matter tracts of the olfactory system.
\end{abstract}

Conclusion: The DTI technique is a powerful tool for obtaining the complete structure of white matter associated with the olfactory system which allows connections between different areas of the brain to be explored. The results of DTI show white matter maturation as a function of age, and this could be used to characterize changes in white matter density and its organization.

\section{Keywords}

DTI, Olfactory, MRI, Tractography

\section{Introduction}

Magnetic resonance imaging (MRI) is a powerful tool capable of visualizing the anatomy of tissues in vivo non-invasively. MRI uses non-ionizing radiation, which makes it a fairly safe technique for which no negative effects on tissues have been demonstrated [3].

We have used the magnetic resonance technique known as diffusion tensor imaging (DTI), a relatively new modality of MRI which provides information on the degree and directionality of water diffusion in tissues and on microstructural brain development [4].

DTI relies on the Brownian motion of water molecules within tissue. The movement of water molecules

Citation: Ramírez-Flores H, Barragan-Perez E, Dies-Suarez P, Platas-Neri D, Garcia J, et al. (2019) Characterization of the Olfactory Pathway by Anisotropic Diffusion Using Nuclear Magnetic Resonance Imaging in a Pediatric Population. Int J Radiol Imaging Technol 5:057. doi.org/10.23937/2572-3235.1510057 Accepted: December 07, 2019: Published: December 09, 2019

Copyright: (c) 2019 Ramírez-Flores H, et al. This is an open-access article distributed under the terms of the Creative Commons Attribution License, which permits unrestricted use, distribution, and reproduction in any medium, provided the original author and source are credited. 
is dependent on both the microscopic and macroscopic restrictions of the tissue.

Brain functions require efficient communication networks between different regions of the brain and between the brain and the rest of the body. These functional networks can be modulated by brain white matter (WM) tissues [5]. White matter consists mainly of densely clustered nerve fibers, each cluster is comprised of an axon extending from the neuron body with a long, narrow cylindrical geometry and surrounded by myelin [6]. The main function of axons is to drive electrical signals between neurons. This signal is reinforced by myelin and other biological processes, which accumulate around the axons as brain development takes place. Myelin consists of concentric layers of lipids and proteins that isolate the axon while providing mechanical and biochemical support [7].

Within the brain WM, water molecules tend to dissipate more freely along the direction of the axonal fascicles than across them. The diffusion parameters that can be obtained are therefore modulated by the tissue barriers. This directional dependence of diffusivity is called anisotropy [8]. The two main parameters derived from DTI data are the apparent diffusion coefficient (ADC) and the fractional anisotropy (FA). FA reflects the directionality of molecular displacement by diffusion and varies between " 0 " (isotropic diffusion) and " 1 " (infinite anisotropic diffusion). ADC reflects the average magnitude of molecular displacement by diffusion; the higher the ADC value [9], the more isotropic the medium. It is possible to obtain 3D maps of brain WM using the DTI technique called tractography, in which a coded color map is overlaid on the images, the color showing the privileged direction of water molecule diffusion [10]. If the direction of the diffusion is from left to right, it is assigned a red color. If the direction is from bottom to top, it is assigned a blue color. Lastly, if the direction of the diffusion is from the posterior to the anterior part, it is assigned a green color. Tractography is a non-invasive technique that is already used as a tool that permits surgical planning in brain tumors [11].

The olfactory sense plays an important role in everyday life. However, neither the anatomy of the olfactory pathway nor its development in child populations have been studied in depth.

Whilst the anatomy of the olfactory structure has previously been investigated using dissection microsurgery [12], the use of magnetic resonance tractography makes it possible to visualize the complicated olfactory system non-invasively.

The olfactory pathway begins with the olfactory receptors embedded in a specialized mucous membrane in the upper part of the nasal cavity [13]. The receptors are bipolar neurons covered with modified, non-motile cyclins, which probably contain the active sites involved in the process of olfactory transduction. Axons from the olfactory receptors enter small nerve bundles that pass through the perforations in the cribriform plate of the ethmoidal bone and enter the olfactory bulbs.

Olfactory bulbs are found in the ventral aspect of the frontal lobes [14]. The olfactory bulbs and all other parts of the olfactory pathway are telencephalic derivatives. Within the olfactory bulbs, the olfactory nerves synapse in the mitral cells, whose axons project directly into the olfactory cortex.

The olfactory cortex is located at the base of the frontal lobe and medial aspect of the temporal lobe. on which the anterior perforated substance, through which the striated arteries enter the inside of the brain, is superimposed. In the temporal lobe, the olfactory cortex covers the rostral portion of the Para hippocampal gyrus, including a median protuberance known as a single or uncinate gyrus.

From the olfactory cortex, olfactory information is transmitted through the medio dorsal nucleus of the thalamus to the insular and orbitofrontal cortex. The insular cortex, which is buried in the depths of the Sylvian fissure, also receives taste input from the medial part of the Ventral Posterolateral Nucleus (VPM) and is believed to be the site where olfactory and taste information is integrated to produce the sensation known as flavor.

The orbitofrontal cortex at the base of the frontal lobe plays an unknown role in olfactory perception.

The purpose of this study is to characterize the entire white matter tracts corresponding to the olfactory system, and evaluate the differences in diffusion parameters as a function of age in healthy Mexican children.

\section{Materials and Methods}

\section{Patients}

Forty-seven children (ages 6-14 with a standard deviation of \pm 2.25 ) were examined, 17 girls and 30 boys with an average age of 10.32 , without any apparent neurological or psychiatric disease. The study was approved by the Ethical and Research Committee of the Hospital Infantil de Mexico, Federico Gomez, and according to the International Principles of the Ethical Treatment of Children in Research.

\section{Obtaining images}

Experiments were performed in a 1.5 T Philips Intera Achieva scanner (Philips, Inc., Netherlands) using an 8 channel SENSE head-coil and Gradient Coils NOVA, (Copley 271 Dual, slew rate $80 \mathrm{mT} / \mathrm{m} / \mathrm{ms}$, peak amplitude $120 \mathrm{mT} / \mathrm{m}$ ) covering the entire brain. DTI imaging was performed with an axial EPI sequence with settings TR = $9491 \mathrm{~s}, \mathrm{TE}=75 \mathrm{~ms}, \mathrm{FOV}=230 \mathrm{~mm} \times 230 \mathrm{~mm}$, matrix 124 


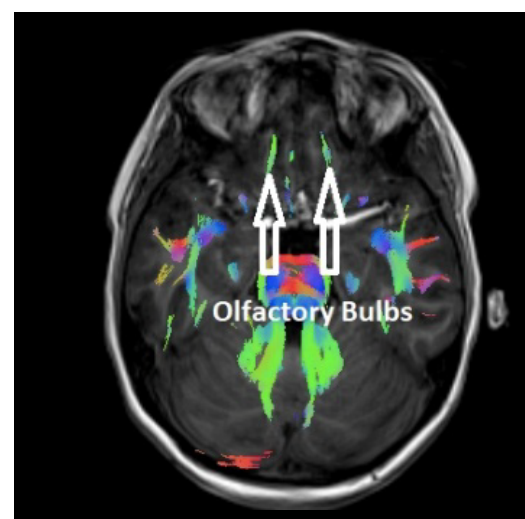

a)

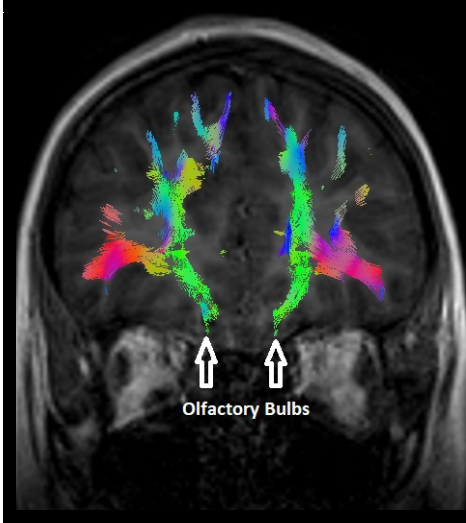

c)

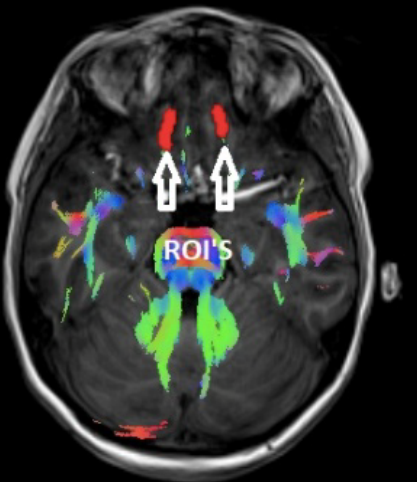

b)

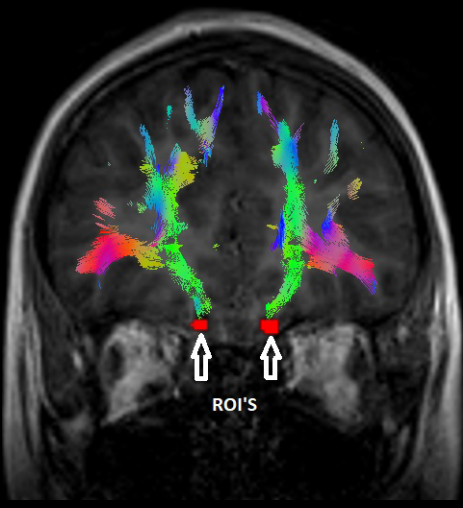

d)

Figure 1: ROIs on the olfactory bulbs where information about odours. a) Axial view of the tracts corresponding to the olfactory bulbs, b) Axial view of the ROls placed on the olfactory bulb, c) Coronal view of the tracts corresponding to the olfactory bulbs and d) Coronal view of the ROIs on the olfactory bulb.

$\times 124,15$ non-collinear directions (b-value $800 \mathrm{~s} / \mathrm{mm}^{2}$ ) with fat suppression. 3D T1 weighted high resolution images were obtained as anatomical reference with the parameters: $\mathrm{TR}=25 \mathrm{~s}, \mathrm{TE}=4.096 \mathrm{~s}$, slice thickness $=1.6$ $\mathrm{mm}$, acquisition matrix $=272 \times 272$, Percent phase field of view $=100$, gap $=0.8 \mathrm{~mm}$, flip angle $=30$ ).

Diffusion image analysis was performed using The Oxford Center for Functional Magnetic Resonance Imaging (FMRIB) software, FSL 3.2.0 (https://fsl.fmrib.ox.ac.uk/fsl/fslwiki) applying a reorientation of the images and a correction for eddy currents. The diffusion tensor imaging (DTI) was analyzed using MedINRIA 3.0 software (https://med.inria.fr/), with an FA threshold between 300 , and 200 and a maximum length of 10 . The diffusion tensors were calculated after a correction for movement to obtain generalized FA and ADC values. The segmentation of the different regions was drawn manually in the 3D images with weighting in T1. The tracts obtained were approved by an ethical review group of the Federico Gómez Children's Hospital of Mexico. The functional pathways were segmented according to the literature [15].

\section{Segmentation of the olfactory pathway}

MedINRIA software allows the segmentation of the olfactory tracts by placing ROIs on the tracts of interest with a deterministic tracking algorithm [16], which makes it possible to isolate the tracts of interest from the total tracts in the human brain. In order to segment this functional pathway, ROls were placed at the level of the base of the frontal lobe, where the olfactory bulbs are located [17] (Figure 1).

The MedINRIA software followed tracts corresponding to the limbic system, specifically tracts of the circulated gyri, for which it was necessary to delimit the tracts associated with the olfactory path using regions of avoidance (ROA's) placed on the cingulated gyri and the corpus callosum, which, combined with the ROI's, made it possible to delimit the regions associated with the olfactory pathway.

In this way, the olfactory path corresponding to the tracts of the olfactory bulbs, the olfactory cortex and the orbitofrontal cortex of the brain were characterized. The objective of being able to visualize the WM tracts in a 3D image is to be able to visualize the connectivity between the different brain zones corresponding to the olfactory system, in addition to representing an advantage for the doctor and their corresponding clinical interpretation. The measure of the diffusion parameters of the total tracts that the MedInria software provides (FA and ADC) were mea- 


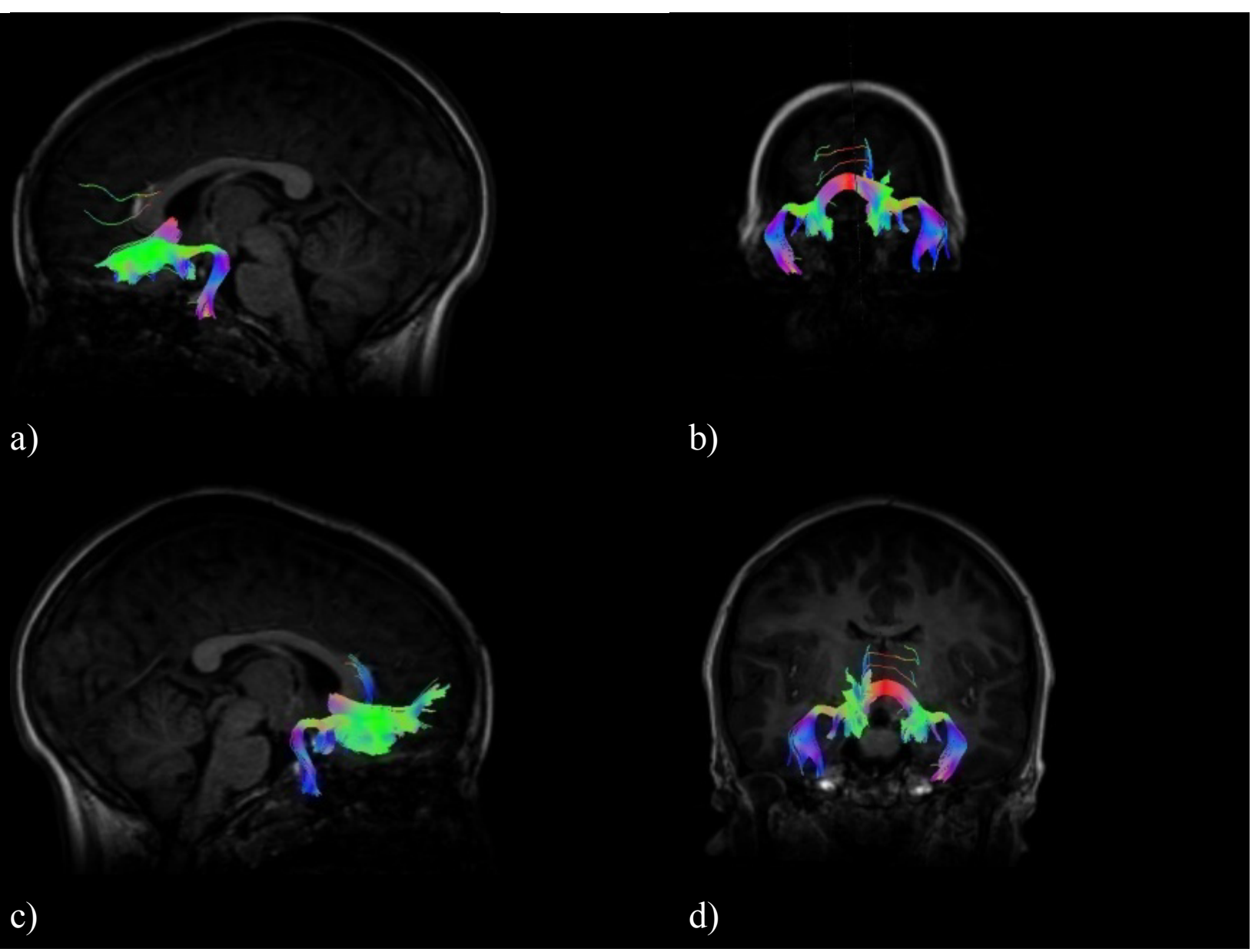

Figure 2: Olfactory pathway tracts containing the olfactory bulbs, the olfactory cortex and the orbitofrontal cortex of the brain, a) Left sagittal view, b) Posterior coronal view, c) Right sagittal view and d) Anterior coronal view.

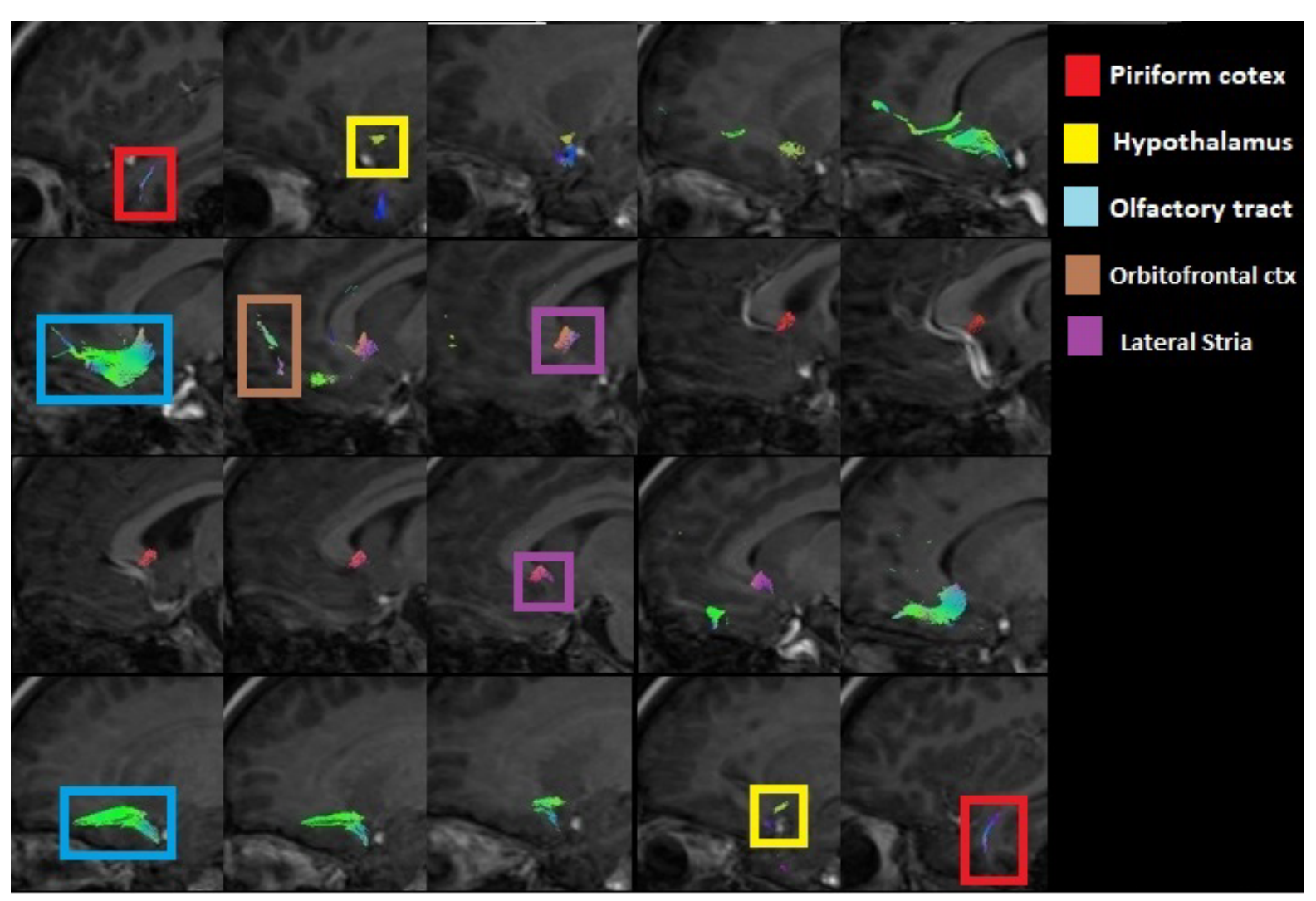

Figure 3: Sagittal view of the olfactory tracts (left to right) showing the tracts corresponding to the piriform cortex, the hypothalamus, the olfactory tract from the olfactory bulbs, the orbitofrontal cortex and the lateral stria. 
sured; these tracts can be seen in (Figure 2).

In all the subjects, it was possible to reconstruct the WM tracts forming the olfactory pathway in a similar way.

\section{Lateral Stria}

The olfactory tracts obtained are projected directly towards the piriform lobe located at the level of the hippocampus in the temporal cortex in the frontal part

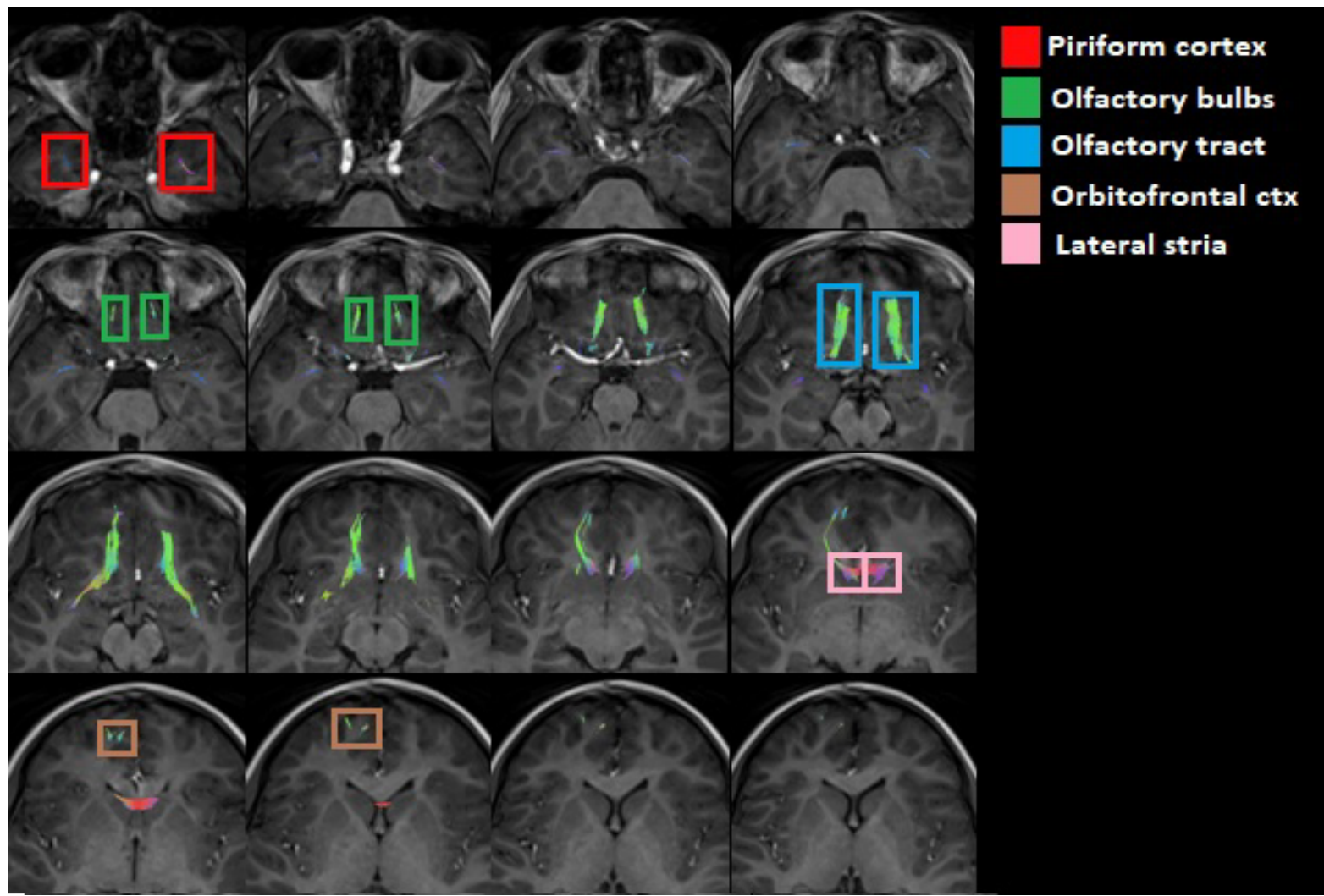

Figure 4: Axial view of the olfactory tracts (inferior to superior) emphasizing the piriformis cortex, the olfactory bulbs, the olfactory tract of the olfactory bulbs, the orbitofrontal cortex and the lateral stria.

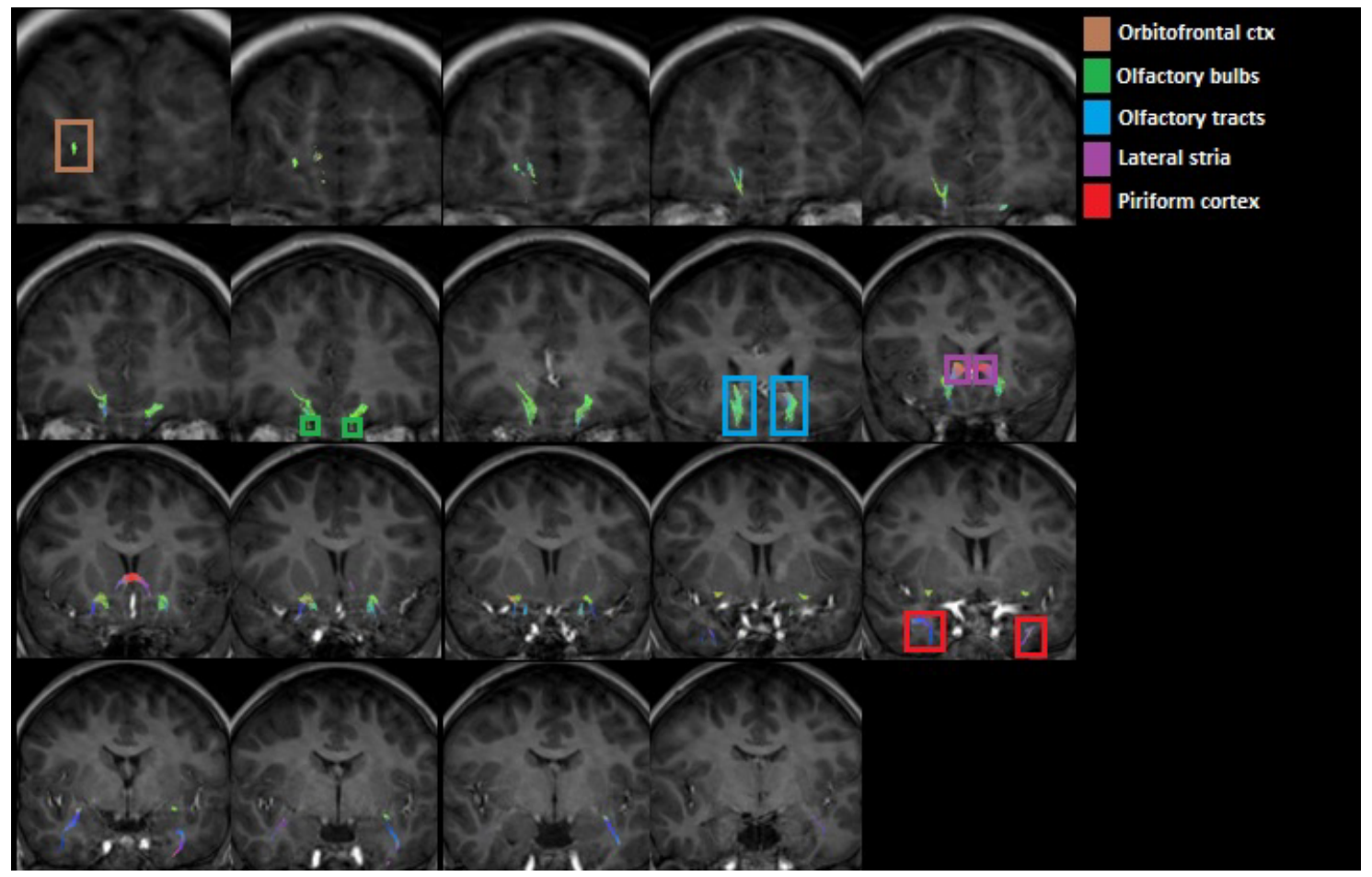

Figure 5: Coronal view of the olfactory tracts (anterior to posterior) emphasizing the piriformis cortex, the olfactory bulbs, the olfactory tract of the olfactory bulbs, the orbitofrontal cortex and the lateral stria. 
of both hemispheres (Figure 3, Figure 4 and Figure 5). There are tracts between the amygdala and the olfactory tract.

\section{Direct and indirect olfactory pathways}

There are tracts that connect the piriform lobe with the orbitofrontal cortex: The indirect way that passes through the thalamus, which is involved in the perception and discrimination of smells, and the fibers that surround the temporal lobe of the lateral ventricle and run through the sub lenticular white matter (Figure 3,
Figure 4 and Figure 5).

\section{Results}

The FA and the ADC of the complete system were measured, giving the generalized FA and ADC values.

Figure 6 shows the values obtained for the generalized FA diffusion parameter, whose range of values is between ( 0.307 a.u.) at the age of 11 and reaches a value of (0.461 u.a.) at the age of 11 , with an average of (0.387 a.u.).

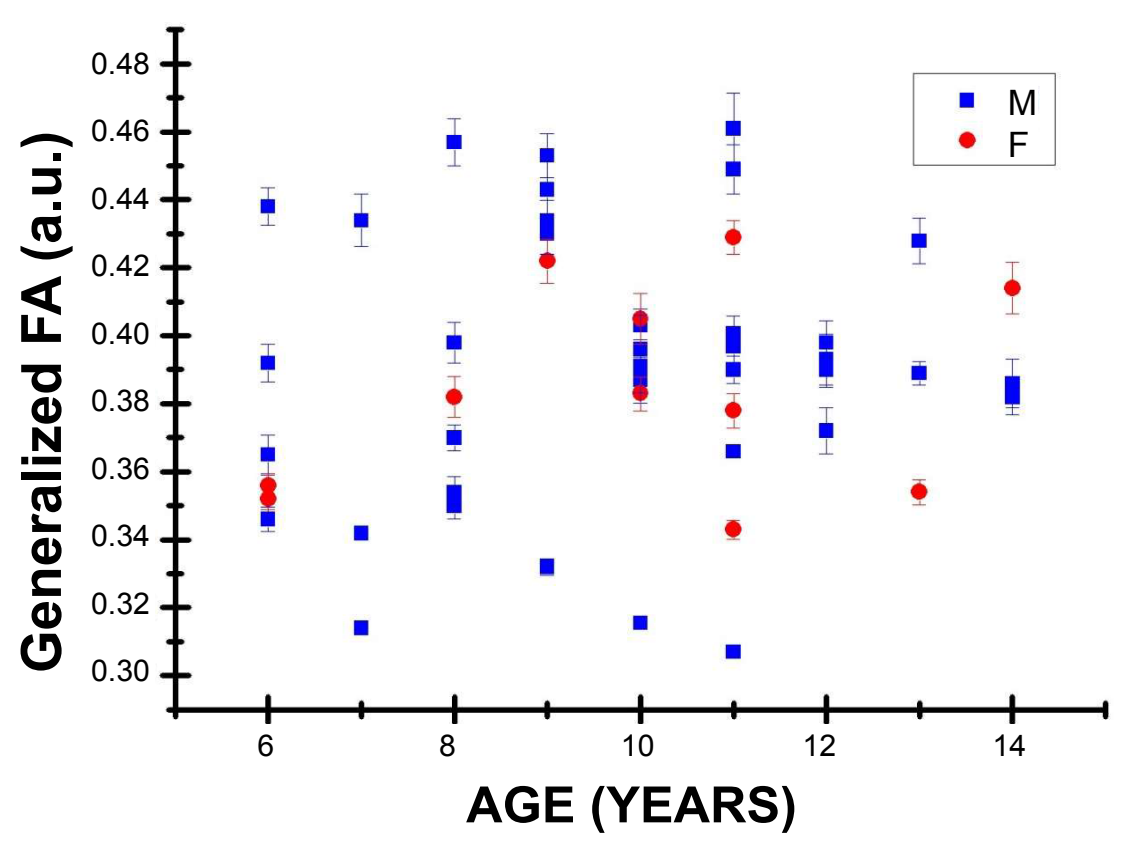

Figure 6: Generalized Fractional Anisotropy values in arbitrary units ranging from 0 to 1 ; F for female and $M$ for male children.

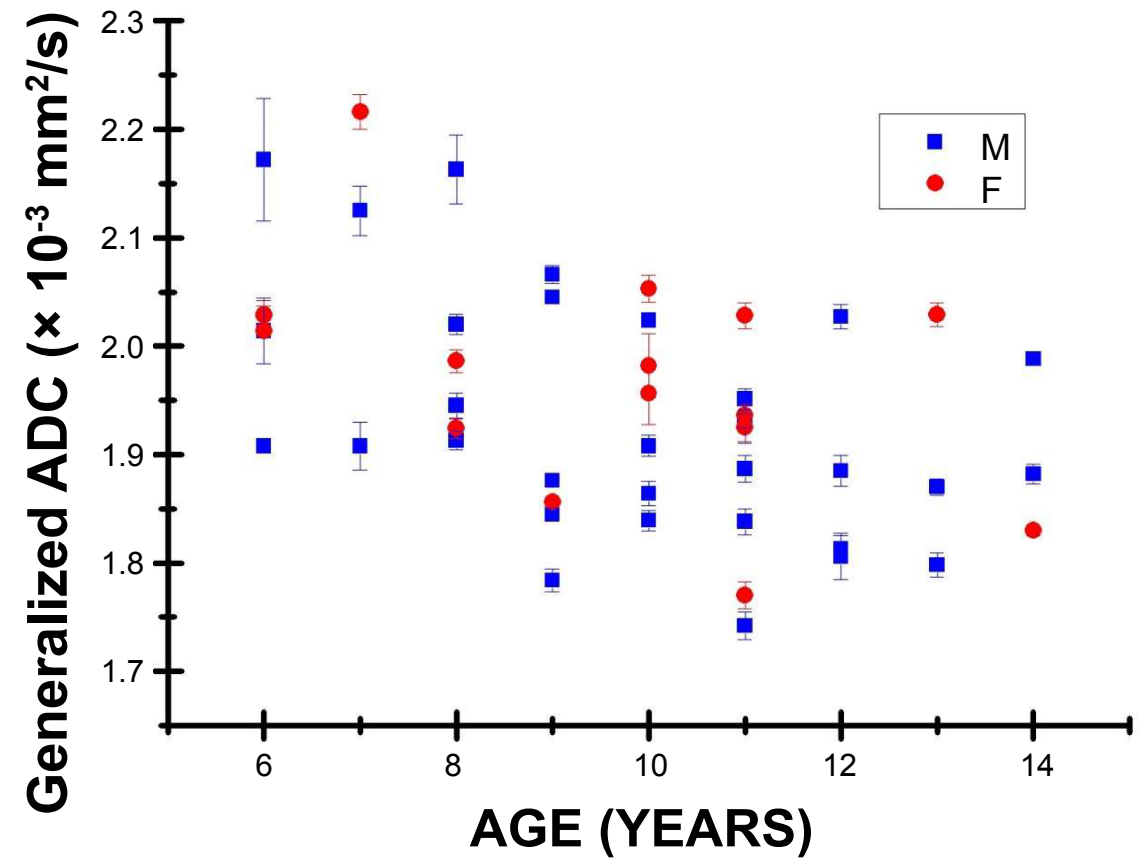

Figure 7: Values of the Generalized Apparent Diffusion Coefficient, where an emphasis has been placed on the sexes of children. 
Table 1: Coefficient of correlation between age and the generalized diffusion parameter values.

\begin{tabular}{|l|l|l|}
\hline \multicolumn{1}{|l|}{} & \multicolumn{2}{l|}{ Age } \\
\hline Generalized FA & r & p \\
\hline Generalized ADC & 0.192 & 0.177 \\
\hline & -0.503 & 0.000 \\
\hline
\end{tabular}

r: Pearson coefficient; P: Statistically significant $p \leq 0.05$.

Figure 7 shows the values of the generalized ADC diffusion parameter, with a range of values between $\left(1.742 \mathrm{~mm}^{2} / \mathrm{s}\right)$ at the age of 11 and $\left(2.216 \mathrm{~mm}^{2} / \mathrm{s}\right)$ at the age of 7 , with an average of $\left(1.944 \mathrm{~mm}^{2} / \mathrm{s}\right)$.

The corresponding statistics were obtained using the SPSS software ("https://www.ibm.com/mx-es/analytics/spss-statistics-software"), seeking a Pearson correlation coefficient between the age variable and the values of the diffusion parameters respectively generalized with a statistical significance of $p<0.05$.

Table 1 contains the results for the statistics and their corresponding Pearson correlation coefficients. No significant statistic was found relating the value of generalized FA and the age of the patients. Conversely, for the generalized ADC, a significant statistic was found for the age of the patients.

\section{Discussion of Results}

$A D C$ values are predominantly caused by the orientation of fiber tracts in white matter and are influenced by their micro- and macrostructural characteristics as well as the magnitude of the diffusion (of the water molecules) within the tissue [18].

Figure 7 shows that the generalized ADC values decrease as age increases, with a moderate significant Pearson correlation coefficient, showing that as the brain develops, the value of ADC decreases. In other words, the molecules of water contained in the white matter of the brain acquire a more specialized direction of diffusion that is probably related to the process of myelination of the axons between neurons [19], among other physiological processes that may influence this process.

Figure 6 shows that the values of generalized FA tend to increase with age; nevertheless, a sufficiently significant Pearson correlation coefficient was not found. This diffusion parameter is also affected by the process of myelination, axon density and other processes in white matter axons in the brain [20]. The process of myelination occurs rapidly in the first two years of neural development, although suggestions have been made that this process continues until early adulthood [21,22]. These previous studies suggest that the amount of myelin (and other WM properties) continues to increase during neurological development and this is reflected in an increase in the volume of white matter, and the resulting in- creased myelination and axon thickness contribute to functionality development by increasing conduction speed [23].

The observed structural data reflect the classic neuroanatomical descriptions, now enhanced with the ADC and FA measurements, which provide quantitative measures that can be used in comparative studies obtaining control values by age and sex that can be compared with sick patients and can be used as a tool for clinical results.

\section{Conclusions}

Generalized FA and ADC values can be seen to change with age. According to existing understanding about the development of the brain, the degree of myelination varies according to age, although previous studies suggest that the myelination process develops mostly in the first 5 years [24]. The neurological maturation process may also depend on the diameter of the axons and thickness of the insulated myelin sheath [25]. It is therefore useful to evaluate the normal development of children's brains. The results obtained have some similarity to those obtained by previous studies on different areas of the brain [26].

The lack of knowledge on the olfactory pathway makes this functional area a particularly important area to study, especially in pediatric populations. This can open the door to the early detection of diseases related to this pathway among the wider population, not just among pediatric population, such as Parkinson's [27] and other diseases affecting the neurological development process.

The olfactory sense has a physiological influence in aspects of everyday life in a number of domains of human behavior such as attention [28], emotions [29], memory [30] and visual-motor interaction.

The use of the DTI imaging technique, with its capacity to discern the orientation of white matter tissues and to reconstruct their structure in 3D, has opened the door to selective brain studies in healthy and diseased human brains. Being able to extract several diffusion parameters will make it possible to study the normal development of the brain and its specialization of tracts to obtain control values for diffusion parameters and compare them with those of sick patients.

\section{Acknowledgment}

Technologists Manuel Obregon and Porfirio Ibanez.

\section{References}

1. Mori S, Zhang J (2006) Principles of diffusion tensor imaging and its applications to basic neuroscience research. Neuron 51: 527-539.

2. Fadeel SR, Montasser MM, Etaby AN, Darweesh RM (2015) The role of diffusion weighted magnetic resonance imaging in assessment of normal myelination in infantile 
brain. Alexandria Journal of Medicine 51: 271-276.

3. Dempsey MF, Condon B, Hadley DM (2002) MRI safety review. Semin Ultrasound CT MR 23: 392-401.

4. Basser PJ, Mattiello J, LeBihan D (1994) MR diffusion tensor spectroscopy and imaging. Biophys J 66: 259-267.

5. Brown RW, Cheng YCN, Haacke EM, Thompson MR, Venkatesan R (2014) Magnetic resonance imaging: Physical principles and sequence design. John Wiley and Sons.

6. Filley CM, Fields RD (2016) White matter and cognition: Making the connection. J Neurophysiol 116: 2093-2104.

7. Boggs JM (2006) Myelin basic protein: A multifunctional protein. Cell Mol Life Sci 63: 1945-1961.

8. Assaf $Y$, Pasternak $O$ (2008) Diffusion tensor imaging (DTI)-based white matter mapping in brain research: $A$ review. J Mol Neurosci 34: 51-61.

9. Xing D, Papadakis NG, Huang CL, Lee VM, Carpenter TA, et al. (1997) Optimised diffusion-weighting for measurement of apparent diffusion coefficient (ADC) in human brain. Magn Reson Imaging 15: 771-784.

10. Johansen-Berg H, Behrens TE (2013) Diffusion MRI: From quantitative measurement to in vivo neuroanatomy. ( $2^{\text {nd }}$ edn), Academic Press.

11. Ekstrand CL, Mickleborough MJ, Fourney DR, Gould LA, Lorentz EJ, et al. (2016) Pre-surgical integration of fMRI and DTI of the sensorimotor system in transcortical resection of a high-grade insular astrocytoma. Front Integr Neurosci 10: 15 .

12. Kavoi BM, Jameela $H$ (2011) Comparative morphometry of the olfactory bulb, tract and stria in the human, dog and goat. Int J Morphol 29: 939-946.

13. Hawkes C (1995) Handbook of olfaction and gustation. J Neurol Neurosurg Psychiatry 59: 564.

14. Buschhüter D, Smitka M, Puschmann S, Gerber JC, Witt M, et al. (2008) Correlation between olfactory bulb volume and olfactory function. Neuroimage 42: 498-502.

15. R Nieuwenhuys, J Voogd, C Van Huijzen (2008) The human central nervous system. Springer.

16. Fillard P, Toussaint N, Pennec X (2006) Medinria: DT-MRI processing and visualization software. Similar NoE Tensor Workshop 5: 7.

17. Skorpil M, Rolheiser T, Robertson H, Sundin A, Svenningsson $P$ (2011) Diffusion tensor fiber tractography of the olfactory tract. Magn Reson Imaging 29: 289-292.
18. Sener RN (2001) Diffusion MRI: Apparent diffusion coefficient (ADC) values in the normal brain and a classification of brain disorders based on ADC values. Comput Med Imaging Graph 25: 299-326.

19. Klingberg T, Vaidya CJ, Gabrieli JD, Moseley ME, Hedehus $M$, et al. (1999) Myelination and organization of the frontal white matter in children: A diffusion tensor MRI study. Neuroreport 10: 2817-2821.

20. Basser PJ, Pierpaoli C (2011) Microstructural and physiological features of tissues elucidated by quantitative-diffusion-tensor MRI. J Magn Reson 213: 560-570.

21. Yakovlev P, Lecours AR (1967) The myelogenetic cycles of regional maturation of the brain. Regional development of the brain in early life. 3-70.

22. Martin AR, Aleksanderek I, Cohen-Adad J, Tarmohamed Z, Tetreault $L$, et al. (2016) Translating state-of-the-art spinal cord MRI techniques to clinical use: A systematic review of clinical studies utilizing DTI, MT, MWF, MRS, and fMRI. Neuroimage Clin 10: 192-238.

23. Ritchie JM (1984) Physiological basis of conduction in myelinated nerve fibers. In: Morell P, Myelin. Springer, Boston, MA, 117-145.

24. Nakagawa $H$, Iwasaki S, Kichikawa K, Fukusumi A, Taoka T, et al. (1998) Normal myelination of anatomic nerve fiber bundles: MR analysis. AJNR Am J Neuroradiol 19: 11291136.

25. Aboitiz F, Scheibel AB, Fisher RS, Zaidel E (1992) Fiber composition of the human corpus callosum. Brain Res 598: 143-153.

26. Schmithorst VJ, Wilke M, Dardzinski BJ, Holland SK (2002) Correlation of white matter diffusivity and anisotropy with age during childhood and adolescence: A cross-sectional diffusion-tensor MR imaging study. Radiology 222: 212218.

27. Georgiopoulos C, Warntjes M, Dizdar N, Zachrisson H, Engström M, et al. (2017) Olfactory impairment in Parkinson's disease studied with diffusion tensor and magnetization transfer imaging. J Parkinsons Dis 7: 301-311.

28. Keller A (2011) Attention and olfactory consciousness. Front Psychol 2: 380.

29. Homma I, Masaoka Y (2008) Breathing rhythms and emotions. Exp Physiol 93: 1011-1021.

30. Wilson DA, Best AR, Sullivan RM (2004) Plasticity in the olfactory system: Lessons for the neurobiology of memory. Neuroscientist 10: 513-524. 